\title{
Vascular Area with Marked Resistance Elevation in One-clip, Two-kidney Renovascular Hypertensive Rats
}

\author{
Yasuhiro TeRANISHI and JURo IRIUCHIJIMA \\ Department of Physiology, School of Medicine, University of Hiroshima, \\ Hiroshima, 734 Japan
}

\begin{abstract}
Blood flow in the terminal aorta or superior mesenteric artery was recorded in conscious one-clip, two-kidney renovascular hypertensive rats and normal control rats with a chronically implanted electromagnetic flow probe. An indwelling catheter for direct measurement of arterial pressure was inserted into the terminal aorta in rats with a flow probe around the superior mesenteric artery. In the hindquarter area supplied by the terminal aorta, the elevation of vascular resistance in hypertensive rats in relation to normal rats was close to the average over the whole body. However, it was about $40 \%$ more intense than the average in the superior mesenteric area. Quantitatively, the hindquarter and superior mesenteric beds in hypertensive rats contributed about a quarter and a third of the decrease in total vascular conductance, respectively. Plots of superior mesenteric flow per body weight against arterial pressure at rest for 51 measurements in 9 hypertensive rats revealed an inverse relation between these two variables. These findings indicate that elevation of vascular resistance in the superior mesenteric area and probably the splanchnic area in general play an important role in one-clip, twokidney renovascular hypertension.
\end{abstract}

Key words: renovascular hypertension, peripheral resistance, splanchnic flow, muscle flow.

Since arterial pressure is a product of total peripheral resistance and cardiac output, hypertension should be due to increase of total peripheral resistance and/or cardiac output. However, most hypertensive states are ascribable to increase in total peripheral resistance rather than cardiac output (FREIS, 1960; OLMSTED and PAGE, 1965). In one-clip, two-kidney renovascular hypertensive rats, too, direct measurement of cardiac output in the conscious resting state with an electromagnetic flow probe chronically implanted around the ascending aorta revealed a normal cardiac output with an elevated total peripheral resistance to maintain the hypertension (TERANISHI and IRIUCHIJIMA, 1983).

Received for publication July 18, 1984

寺西泰弘, 入内島十郎 
The next question regarding the hemodynamic state of this experimental hypertensive model is whether the elevation of resistance is uniformly distributed in various peripheral vascular beds or, alternatively, resistance is elevated especially markedly in some vascular beds. In the spontaneously hypertensive rat (SHR) (Окамото and АокI, 1963), the elevation of resistance was not uniform, and about $1.5 \times$ more intense in the hindquarter area than the average over the whole body. The decrease in conductance, inverse of resistance, in this area occupied about $40 \%$ of the decrease in the total conductance, inverse of the total peripheral resistance (IRIUCHIJIMA, 1983).

The above conclusion was obtained by observing the peripheral blood flow in various arteries in SHR and normotensive control rats in the conscious state with chronically implanted electromagnetic flow probes. To evaluate the importance of each vascular area as a contributor to the elevation of total peripheral resistance, use was made of two indices, $\alpha$ and $\beta$, calculated from observed values of arterial pressure and flows (IRIUCHIJIMA, 1982).

The same method was applied to one-clip, two-kidney renovascular hypertensive rats in the present study. Using this kind of experimentally hypertensive rats, FLOHR et al. (1976) observed peripheral flow and resistance distribution by the particle distribution method under light anesthesia. However, in view of the wide diversity of results depending on methods and conditions in SHR (ToBIA et al., 1974; NishiYama et al., 1976; Ferrone et al., 1979; KaWAUE et al., 1982; IrIUCHIJIMA, 1983), we considered this study worthwhile.

\section{METHODS}

Preparation of hypertensive rats. Male Wistar rats about 10 weeks of age were anesthetized by intraperitoneal injection of thiamylal sodium at a dose of $50 \mathrm{mg} /$ $\mathrm{kg}$. The right renal artery was reached retroperitoneally by a flank incision and a clip made from a metal tape, $1 \mathrm{~mm}$ wide and $0.1 \mathrm{~mm}$ thick with a gap of $0.3 \mathrm{~mm}$, was placed on it. The contralateral kidney and renal artery were left intact.

Implantation of flow probes and catheters Two to 4 weeks after application of the clip, after confirming the development of hypertension by indirect measurement of arterial pressure by a tail-cuff method, the rats were anesthetized again with thiamylal for chronic implantation of an electromagnetic flow probe and arterial and venous catheters. The tenchnical details have been reported previously (KAWAUE and IrIUChiJima, 1984). In brief, the rat was placed right side down. After a left flank incision, retroperitoneally, a Nihon Kohden electromagnetic flow probe was attached around the terminal aorta (internal diameter of probe: $2 \mathrm{~mm}$ ) or superior mesenteric artery $(1.5 \mathrm{~mm})$. Only one probe was implanted in each rat. The lead wire to the plug from the probe was passed under the skin and led to the dorsal neck to exteriorize the plug through a skin incision.

In rats with a flow probe implanted around the superior mesenteric artery, a 
polyethylene catheter for recording arterial pressure was inserted from the right femoral artery to the terminal aorta. In all rats, a polyethylene catheter for intravenous injection was inserted into the right external jugular vein. The outer end of these arterial and venous catheters, which was plugged with a short piece of stainless steel wire, was led to the dorsal neck and exteriorized. As controls, intact male Wistar rats of similar age were implanted with flow probes and catheters.

Measurement. After probe implantation, the rat was kept separately in a white polyethylene cage of $35 \times 30 \times 17 \mathrm{~cm}$ in size containing wood chips. Water and pellets were given ad libitum. Flow and pressure measurements were begun when more than 2 days had elapsed after implantation and the rat had resumed taking ample water and pellets. The cable from the flowmeter circuit (Nihon Kohden MFV-1100) was connected to the plug of the flow probe and the polyethylene tube from a pressure transducer to the arterial catheter in the rat remaining in the home cage. The cable and tube were both made long enough to allow the rat to move almost freely in the cage during measurement. The carrier amplifier used with the pressure transducer was a Nihon Kohden RP-3. Flow and pressure were recorded with a rectangular pen-writer.

To excite the rat, transposition response (IRIUCHIJIMA et al., 1980) was induced by transposing the rat by hand from its home cage to a new cage made of white polyethylene measuring $30 \times 20 \times 13 \mathrm{~cm}$ and without chips.

Each flow probe was calibrated before implantation by passing known amounts of saline through an excised artery. Flow signals were integrated with a time constant of 0.3 or $1 \mathrm{sec}$ to obtain the mean flow. All flows were normalized to $100 \mathrm{~g}$ of body weight for comparison.

Evaluation of the part played by a vascular area in resistance elevation in hypertension. For this purpose, two indices, $\alpha$ and $\beta$, were computed from flow and pressure values (IrIUCHIJIMA, 1982). By definition

$$
\alpha=(\Delta r / r) /(\Delta R / R),
$$

and

$$
\beta=\Delta g / \Delta G,
$$

where $r, R, g$, and $G$ are the local peripheral resistance of a particular area, total peripheral resistance, peripheral conductance of the area $(1 / r)$, and total conductance $(1 / R)$, respectively, of control normal rats. $\Delta$ refers to the difference between the hypertensive and normal rats. Group mean flow and pressure values were used in the computation of these indices. The larger the $\alpha$, the greater was the elevation of the resistance of the particular vascular area relative to the average elevation of vascular resistance over the whole body. $\beta$ indicates how much the percentage of the decrease in total conductance is contributed by the particular area.

Statistical analysis. Student's $t$-test was used throughout. 


\section{RESULTS}

Hindquarter flow measured in terminal aorta

Since only this flow is significantly smaller in SHR than in normal rats and since the elevation of resistance in this vascular area in SHR plays an important role in their hypertension (IRIUCHIJIMA, 1983), our attention was first paid to this vascular area.

The record reproduced in Fig. 1 shows a successive change in hindquarter flow at the terminal aorta in a hypertensive rat over a period of a few minutes. The blood pressure measured indirectly at the tail prior to implantation of the flow probe was $180 \mathrm{mmHg}$. Near the letter $\mathrm{Q}$ in the figure the rat was apparently quiet. At around $T$ the rat was grasped by the experimenter's hand and transposed to the new cage to induce transposition response. After the flow had reached a new plateau level, the rat was grasped again and returned to the home cage at around $\mathrm{B}$.

The relatively steady flow level as at $Q$ in the figure was noted as the resting flow. In each rat, several resting flow values were measured and averaged to obtain the value representing the particular rat. Then the group mean and S.D. were calculated from the flow values representing each rat. The mean hindquarter flow \pm S.D. from 9 hypertensive rats (body weight: $352 \pm 40.0 \mathrm{~g}$; tail blood pressure

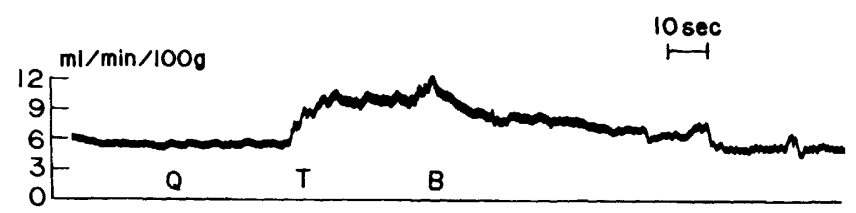

Fig. 1. Hindquarter flow observed in a conscious one-clip, two-kidney renovascular hypertensive rat with a chronically implanted electromagnetic flow probe around the terminal aorta. At $\mathrm{Q}$ the rat was apparently at rest. It was transposed to a new cage to induce transposition response at $T$ and returned to the home cage at $B$.

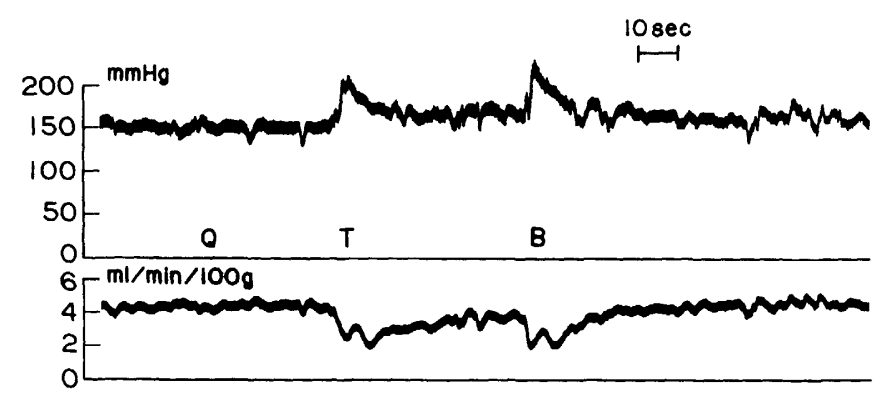

Fig. 2. Arterial pressure (top) and superior mesenteric flow (bottom) observed in a conscious one-clip, two-kidney renovascular hypertensive rat. $Q$, quiet; $T$, transposed to a new cage; $\mathrm{B}$, back to the home cage. 
before implantation: $182 \pm 21.5 \mathrm{mmHg})$ was $5.31 \pm 0.68 \mathrm{ml} /(\mathrm{min} \cdot 100 \mathrm{~g})$. The corresponding value from 11 normal control rats (body weight: $296 \pm 42.9 \mathrm{~g}$ ) was $5.34 \pm 0.77 \mathrm{ml} /(\mathrm{min} \cdot 100 \mathrm{~g})$ and the difference of the means was statistically insignificant.

\section{Superior mesenteric flow}

Figure 2 represents one example of simultaneous recording of arterial pressure and superior mesenteric flow in a hypertensive rat. Transposition response was induced as with hindquarter flow (Fig. 1). When the rat was grasped by hand for transposition, the superior mesenteric flow was decreased markedly and the arterial pressure was increased. After releasing the rat to a new cage, the flow and pressure gradually recovered partially and reached new plateau levels, which were noted as the flow and pressure during the transposition response. During the response, the flow was markedly less and the pressure was slightly higher than before.

The mean superior mesenteric flow and other parameters \pm S.D. at rest from 9 hypertensive rats and 13 normal control rats are presented in Table 1. The mean superior mesenteric flow was significantly smaller in the hypertensive rats than in the controls.

In Fig. 3, resting superior mesenteric flow is plotted against arterial pressure in a total of 51 measurements in 9 hypertensive rats (filled circles) and 20 measurements in 13 normal rats (open circles). Flow was inversely related to pressure in hypertensive rats $(r=-0.553, p<0.001)$ but not in normal rats.

\section{$\alpha$ and $\beta$ indices}

Calculated $\alpha$ and $\beta$ indices for the superior mesenteric and hindquarter vascular beds are presented in Table 2. For the superior mesenteric bed the mean pressure and flow values in Table 1 were used for calculation. Since arterial pressure was not recorded in rats with a probe around the terminal aorta, the mean pressure in Table 1 was also used to calculate the indices for the hindquarter area. The equality of cardiac output between hypertensive rats and normal rats observed in a

Table 1. Comparison of superior mesenteric flow and other parameters between one-clip, two-kidney renovascular hypertensive rats and normal rats.

\begin{tabular}{lccl}
\hline & $\begin{array}{c}\text { Hypertensive rats } \\
(n=9)\end{array}$ & $\begin{array}{c}\text { Normal rats } \\
(n=13)\end{array}$ & $p<$ \\
\hline Arterial pressure $(\mathrm{mmHg})$ & $166 \pm 20.5$ & $108 \pm 7.98$ & 0.001 \\
Heart rate (beats/min) & $369 \pm 22.0$ & $357 \pm 31.3$ & N.S. \\
Superior mesenteric flow $(\mathrm{ml} /(\mathrm{min} \cdot 100 \mathrm{~g}))$ & $4.01 \pm 0.53$ & $5.17 \pm 0.44$ & 0.001 \\
$\quad$ Superior mesenteric resistance & $42.1 \pm 7.99$ & $20.9 \pm 2.16$ & 0.001 \\
$\quad(\mathrm{mmHg} \cdot \min \cdot 100 \mathrm{~g} / \mathrm{ml})$ & $316 \pm 25.6$ & $295 \pm 33.7$ & \\
Body weight $(\mathrm{g})$ & & \\
\hline
\end{tabular}

Mean \pm S.D. 


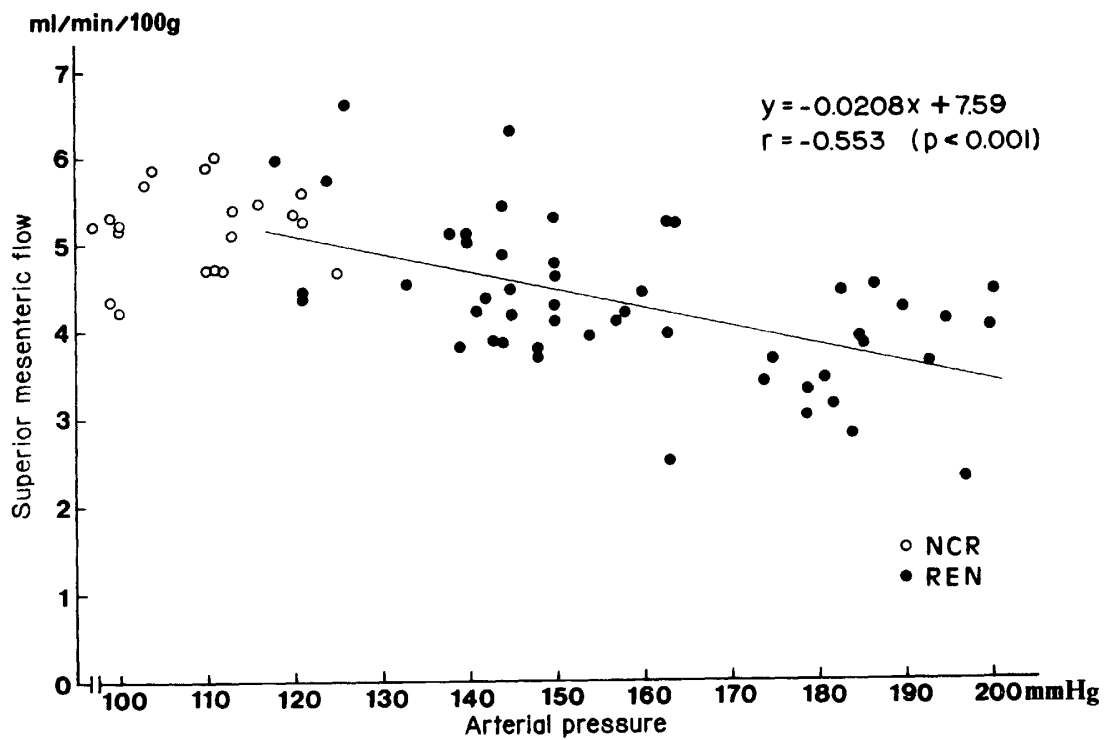

Fig. 3. Plots of superior mesenteric flow against arterial pressure in 51 measurements in 9 hypertensive rats (filled circles) and 20 measurements in 13 normal rats (open circles). Note the inverse relationship between flow and pressure in hypertensive rats $(r=-0.553$, $p<0.001$ ).

Table 2. $\alpha$ and $\beta$ indices of participation and contribution of superior mesenteric and hindquarter areas in one-clip, two-kidney renovascular hypertension based on the data in Table 1 and hindquarter flow values.

\begin{tabular}{lcc}
\hline Artery & $\alpha$ & $\beta$ \\
\hline Superior mesenteric & 1.39 & 0.32 \\
Hindquarter & 0.99 & 0.24 \\
\hline
\end{tabular}

previous study (TERANISHI and IRIUCHIJIMA, 1983) was utilized in calculating $\alpha$ indices. In calculation of $\beta$ indices, the mean cardiac output for the control rats was assumed to be $22.5 \mathrm{ml} /(\mathrm{min} \cdot 100 \mathrm{~g})$, which was the mean value for the control rats in the same study.

\section{Changes in transposition response}

Percent changes in various parameters in transposition response in hypertensive and normal rats are presented in Table 3. There was no significant difference in any parameter between the two groups of rats.

\section{DISCUSSION}

Unlike SHR in which elevation of vascular resistance is especially marked in the hindquarter area (IRIUCHIJIMA, 1983), the vascular area with marked resistance 
elevation in one-clip, two-kidney renovascular hypertensive rats was found to be the superior mesenteric area. Evaluation with $\alpha$ and $\beta$ indices (Table 2) revealed that here the elevation in vascular resistance was about $1.4 \times$ as intense as the average over the whole body. The decrease in vascular conductance in this vascular bed occupied about one third of the decrease in total conductance. One may further assume that the most important vascular area is the splanchnic bed in this kind of experimental hypertension, while it is the skeletal muscle bed in SHR.

The marked resistance elevation in the splanchnic area in this kind of experimental rat hypertension has been observed by FLOHR et al. (1976) by the particle distribution method under light anesthesia. This consonance between the results obtained by different methods under different conditions may be due to the relatively good stability of renovascular hypertension in contrast to the unstable hypertension in SHR.

As seen from the $\alpha$ index of nearly unity in the hindquarter area in this renovascular hypertensive rat, the elevation of vascular resistance in this area was average. However, since this area drains about a quarter of cardiac output (IRIUCHIJIMA et al., 1982), quantitatively this area contributed also a quarter of the decrease in total conductance, as indicated by the $\beta$ index of about $25 \%$.

Plots of superior mesenteric flow per body weight against arterial pressure showed an inverse relationship between these parameters (Fig. 3). This finding is consistent with the important role assigned to the splanchnic vascular bed in oneclip, two-kidney rat hypertension.

In transposition response when the sympathoadrenal system is excited, cardiovascular changes in SHR were considerably different from those in normal rats. This response was a pressor response in SHR, while it was an isopressor response in normal rats (TADA et al., 1981). The increase in hindquarter flow in the response was far greater in SHR than in normal rats (IRIUCHIJIMA, 1983). However, in one-clip, two-kidney renovascular hypertensive rats, percent changes in various parameters were similar to those in normal rats (Table 3). This is consistent with the generally accepted view that the involvement of the sympathoadrenal system is less in renovascular hypertension than in hypertension in SHR.

Only hindquarter and superior mesenteric flows were observed in the present study. However, these two vascular beds in total drain about $50 \%$ of cardiac out-

Table 3. Percent changes of various parameters in transposition response.

\begin{tabular}{lrr}
\hline Parameter & Hypertensive rats & \multicolumn{1}{c}{ Normal rats } \\
\hline Arterial pressure & $44.4 \pm 6.91$ & $7.09 \pm 7.40$ \\
Heart rate & $20.6 \pm 11.7$ & $15.0 \pm 7.68$ \\
Superior mesenteric flow & $-25.7 \pm 10.5$ & $-23.2 \pm 4.73$ \\
Superior mesenteric resistance & $49.3 \pm 21.0$ & $39.5 \pm 8.95$ \\
Hindquarter flow & $107 \pm 36.8$ & $97.9 \pm 23.9$ \\
\hline
\end{tabular}

Mean \pm S.D. In both groups $n=9$ for all parameters except hindquarter flow for which $n=8$.

Vol. 35, No. 1, 1985 
put in normal rats at rest (IRIUChIJIma et al., 1982). The elevation of vascular resistance in hypertensive rats compared to normal rats was average in the hindquarter area but greater than average in the superior mesenteric area. The decrease in conductance in both areas in total occupied more than $50 \%$ of the decrease in total conductance. Therefore, marked elevation in vascular resistance is unlikely in any other vascular areas except the renal area where resistance elevation is artificially made by clipping the artery.

\section{REFERENCES}

Ferrone, R. A., Walsh, G. M., Tsuchiya, M., and Frohlich, E. D. (1979) Comparison of hemodynamics in conscious spontaneous and renal hypertensive rats. Am. J. Physiol., 236: H403-H408.

Flohr, H., Breull, W., Dahners, H. W., Redel, D., Conradi, H., and Stoepel, K. (1976) Regional distribution of vascular resistance in two models of experimental renovascular hypertension. Pflügers Arch., 362: 157-164.

FreIs, E. D. (1960) Hemodynamics of hypertension. Physiol. Rev., 40: 27-54.

IRIUChIJIMA, J. (1982) Hemodynamics of hypertensive animals. In: Cardiovascular Physiology IV. International Review of Physiology, Vol. 26, ed. by GuYton, A. C. and Hall, J. E. University Park Press, Baltimore, pp. 51-86.

IRIUCHIJMA, J. (1983) Regional blood flow in conscious spontaneously hypertensive rats. Jpn. J. Physiol., 33: 41-50.

IrIUChIJima, J., KaWAUE, Y., and Teranishi, Y. (1982) Blood flow redistribution in the transposition response of the rat. Jpn. J. Physiol., 32: 807-816.

IrIUChiJima, J., Teranishi, Y., and EGUCHI, C. (1980) Transposition response, a cardiovascular response to change of habitat in the rat. Jpn. J. Physiol., 30: 887-896.

KaWAue, Y. and IrIUChiJima, J. (1984) Changes in cardiac ouput and peripheral flows on pentobarbital anesthesia in the rat. Jpn. J. Physiol., 34: 283-294.

Kawaue, Y., Teranishi, Y., and IriuchiJima, J. (1982) Blood flow distribution in anesthetized normal and spontaneously hypertensive rats. Jpn. J. Physiol., 32: 299-301.

Nishiyama, K., Nishiyama, A., and Frohlich, E. D. (1976) Regional blood flow in normotensive and spontaneously hypertensive rats. Am. J. Physiol., 230: 691-698.

Окамото, K. and Aокі, K. (1963) Development of a strain of spontaneously hypertensive rats. Jpn. Circ. J., 27: 282-293.

Olmsted, F. and PAGe, I. H. (1965) Hemodynamic changes in trained dogs during experimental renal hypertension. Circ. Res., 16: 134-139.

TADA, T., Teranishi, Y., and IrIUChIJIMA, J. (1981) Transposition response in different hypertensive rats. Jpn. J. Physiol., 31: 749-752.

Teranishi, Y. and IRIUChiJima, J. (1983) Cardiac output in conscious one-clip, two-kidney renovascular hypertensive rats. Hiroshima J. Med. Sci., 32: 59-63.

Tobia, A. J., Walsh, G. M., TAdepalli, A. S., and LeE, J. Y. (1974) Unaltered distribution of cardiac output in the conscious young spontaneously hypertensive rat: Evidence for uniform elevation of regional vascular resistances. Blood Vessels, 11 : 287-294. 